Journal of Applied Fluid Mechanics, Vol. 2, No. 1, pp. 1-12, 2009.

Available online at www.jafmonline.net, ISSN 1735-3572, EISSN 1735-3645.

DOI: $10.36884 / \mathrm{jafm} .2 .01 .11850$

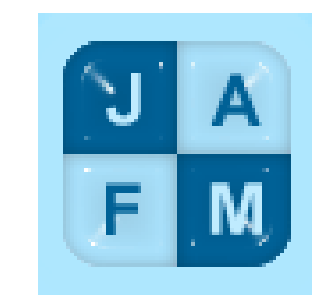

\title{
Experimental and Numerical Approach to Enlargement of Performance of Primary Settling Tanks
}

\author{
A. Razmi ${ }^{1}$, B. Firoozabadi ${ }^{1}$, and G. Ahmadi ${ }^{2}$ \\ ${ }^{1}$ School of Mechanical Engineering, Sharif University of Technology, Azadi Ave., Tehran, Iran \\ ${ }^{2}$ Department of Mechanical and Aeronautical Engineering, Clarkson University, Potsdam, NY 13699, USA.
}

Email: firoozabadi@sharif.edu

(Received August 10, 2007; accepted April 20, 2008)

\begin{abstract}
Circulation regions always exist in settling tanks. These regions would result in short-circuiting enlargement of the dead zone and high flow mixing problems and avoid optimal particle sedimentation. Therefore, the main objective of the tank design process is to avoid formation of the circulation zone, which is known as dead zone. Experiments show that the tank performance can be improved by altering the geometry of the tank which leads to a different velocity distributions and flow patterns. In this paper, the presence of a baffle and its effect on the hydrodynamics of the flow field has been investigated in a primary settling tank. Hydrodynamics of the flow field in these basins is sophisticated. Therefore a numerical simulation has been provided to discover such flow field. Then, the flow structure was simulated by an unsteady two-phase finite volume method, with VOF (Volume of Fluid) model. Besides, the RNG turbulence model was used in the numerical calculations. Successively, in order to validate the mentioned method and for a better understanding of the phenomenon, experimental tests have been carried out using ADV (Acoustic Doppler Velocimeter) and a $0.2 \mathrm{~m}$ breadth rectangular model tank. Results depict the ability of this model in predicting the velocity profiles and circulation districts. Eventually, the optimum position of the baffle for enhancing the performance of the primary settling tank was determined by applying the above mentioned numerical scheme.
\end{abstract}

Keywords: Settling Tank, VOF method, Two-phase flow simulation, Baffle Configuration, ADV

\section{NOMENCLATURE}

$$
\begin{array}{cl}
\text { Fr } & : \text { Froude No., } F r=U / \sqrt{g h} \\
g & : \text { Acceleration of gravity } \\
\mathrm{h}_{0} & : \text { the inlet opening of sluice gate } \\
\mathrm{k} & : \text { turbulent kinetic energy } \\
\mathrm{L} & : \text { Length of the channel } \\
\mathrm{Re} & : \text { Reynolds number, } \mathrm{Re}=U h / \mathrm{U} \\
\mathrm{s} & \text { : baffle location from the inlet }
\end{array}
$$

\section{INTRODUCTION}

Enhancing the efficiency of the settling tanks is one of the most economical approaches for refining water. Experimental and numerical investigations show that the performance of the sedimentation basins is influenced by velocity field variations and the geometry of the tank, especially, the location of the inlet, medium and outlet baffles. Furthermore, the high concentration of the particles leads to the formation of a bottom jet at the bed and reverse- flow on the surface of the tank. It should be noted that the non-uniformity of the velocity field, the short

$\begin{array}{cl}\mathrm{u}_{\mathrm{x}}, \mathrm{u}_{\mathrm{y}} & : \text { Cartesian coordinates of velocity } \\ \mathrm{x} & : \text { distance from the inlet } \\ \alpha_{q} & : \text { Volume fraction of two phases } \\ \varepsilon & : \text { turbulent dissipation energy } \\ \rho & : \text { Fluid density } \\ v & : \text { Kinematic viscosity } \\ \mu & : \text { Fluid viscosity }\end{array}$

circuiting at the surface and the motion of the jet at the bed of the tank which occurs as a result of the circulation in the sedimentation layer, are affected by the geometry of the tank. The modeling of sedimentation basins, which plays a major role in waste-water treatment plants and in a variety of industrial processes separating suspended solids from water, has been vastly investigated by many researchers. As a result of the flow characteristics such as the curvature of the streamlines, the low Reynolds number turbulent flow, and the turbulence anisotropy, some turbulence models may not be successful in predicting such a complex flow filed (Tamayol, Firoozabadi, 2006). 
Sedimentation tanks are divided into two main categories in type and concentration of sludge and particles which are available in them. Primary settling tanks have low influent concentration. Flow field in them is not much influenced by concentration field and buoyancy effects can be negligible. However, secondary or final-settling tanks have higher influent concentration. They usually are placed after primary tanks and activation tanks. So they usually contain activated sludge and as a result of this, size of particles would grow and flow-field is influenced by the concentration distribution.

Various studies have been conducted on effects of a baffle on the flow and hydrodynamics of settling tanks. Brescher et al. (1992) by suggesting velocity and concentration fields for a rectangular clarifier equipped with an intermediate baffle showed that installation of the intermediate baffle was effective. Also, Krebs (1995) investigated the effects of the inlet and intermediate baffles on the flow field in final clarifiers. His research was mainly based on experiments, numerical modeling as well as on analytical relations. But experimental investigations of Taeby-Harandy and Schroeder (1995) on the primary clarifiers showed that the placement of an intermediate baffle, installed close to the middle of the clarifier and extended from the floor upward to one-third depth had no significant effect on the efficiency. They believe that the discrepancy between the result of their studies and the other works is likely due to the difference in the flow patterns meaning that, if the dominant current is a surface current, a baffle extending from the top upward may improve the solid removal efficiency (Taeby-Harandy, Schroeder, 1995).

There are several works were published on the simulation of the settling tanks. Imam et al. (1983) solved the governing equations with a constant turbulent eddy diffusivity assumption. Celik et al. (1985) used the standard $k-\varepsilon$ for turbulence modeling. Adams et al. (1990) and Lyn et al. (1992) used the standard $k-\varepsilon$ turbulence model and the equations obtained from QUICK scheme to predict the turbulent flow field in the settling tanks. They used Flow Through Curves (FTCs) for predicting the performance of the settling tanks. Rodi (1993) investigated the effects of the accuracy of the discretization on the flow field. But Stamou et al. (1990) and many other researchers have shown that obtained results through applying the standard $k-\varepsilon$ model are only qualitatively comparable with experimental data.

Matko et al. (1996) show that empirical models are still widely used today to predict the suspended solids in the effluent and return sludge but cannot model the flow pattern or solids distribution within the tank.

They suggest that the important CFD modeling criteria for the settling of suspended solids in sedimentation tanks are the velocity distribution of the fluid (water in this case), settling velocity distribution of suspended solids, turbulent mass diffusion of suspended solids, and resuspension of settled solids from the tank base. Besides, to give the realistic parameters and to validate these models, experimental data should be entered in these numerical models where possible.
Moreover, work on the analysis of sloshing of water in rectangular open tanks had been extensively carried out by Armenio and La Rocca (1996). Two mathematical models are employed respectively; the Reyolds Averaged Navier Stokes Equations (RANS) and the Shallow Water Equations (SWE).

The RANS were solved using a modified form of the well established MAC method (SIMAC) able to treat both the free surface motion and the viscous stresses over the rigid walls accurately. The Shallow Water Equations were solved by using a simple and powerful algorithm (CEDE) able to deal with large impacting waves over the tank walls. Experimental tests had been carried out using a $0.5 \mathrm{~m}$ width rectangular tank in periodic roll motion.

It had been shown that RANS provide more accurate solutions than SWE for small or moderate amplitudes of excitation. Finally a simple baffle configuration inside the tank had been considered. By the analysis of numerical results, it had been observed that the presence of a vertical baffle at the middle of the tank dramatically changes the sloshing response compared to the unbaffled configuration. It produces a jump-like effect, resulting in a weak magnification of the dynamic loads on the vertical walls out of resonance, and a strong reduction of the dynamic loads in the resonance condition.

Ashjari and Firoozabadi (2003) used the nonlinear $k-\varepsilon$ and FTC for prediction of the flow and tank performance. Using FTC, Tamayol et al. (2006) studied the effects of different inlet positions on the flow field and the efficiency of the settling tanks.

Besides turbulence model, another challenging problem in settling tanks is using the symmetry boundary condition. In the previous studies, the symmetry condition has been applied to the free surface of settling basins that includes zero gradients and zero fluxes perpendicular to the boundary (Tamayol et al., 2007). Then, the effects of the wind and small ripples on the flow-field have been neglected. Since the upper boundary is a free surface, using symmetry boundary condition and a lid approximation in simulation of a settling tank can provide a significant error on the results.

In this study, the effects of the baffle position on the performance of a settling tank have been investigated experimentally and the data were used to verify the numerical model. In the laboratory experiments, the velocity components are measured using Acoustic Doppler Velocimetery (ADVs). Acoustic backscattering measurement is a non-intrusive technique for determining the sediment concentration with a high degree of temporal and spatial resolution (Hay, 1983; Libicki et al., 1989). Acoustic backscattering measurements of suspended sediments have been used in marine, ocean, open channel flows, and fluvial environments (Fugate, Fredrichs, 2002). In the numerical simulation, VOF method which is a powerful tool to determine the morphology and raise characteristics of the hydrodynamic of fluid at the free surface was used. 


\section{EXPERIMENTAL FACILITIES}

The experiments were conducted in a rectangular channel with and without baffles. The channel is $\mathrm{L}=8 \mathrm{~m}$ long, $0.6 \mathrm{~m}$ deep, $0.2 \mathrm{~m}$ wide.

The baffle height is $b=8 \mathrm{~cm}$. The inlet flow passes through a sluice gate which its opening is $h_{o}=11.0 \mathrm{~cm}$. s shown in Fig. 1 is the baffle distance from the inlet of the tank. To estimate the actual results, all laboratory data were repeated 6 times.

This research was conducted for three test case studies for the same flow rates and it is equal to $\mathrm{Q}=42$ (lit $/ \mathrm{min})$ in the experiments (Fig. 2). This is the same inlet opening and the same inlet Reynolds number: $\operatorname{Re}_{i n}=3500$. Froud Number in the inlet and in the basin are $\boldsymbol{F r}_{\boldsymbol{i n}}=0.03, \boldsymbol{F r}=0.0056$ for all cases. Case 1 is no baffle and in Cases 2, and 3, a baffle was located in different distance from the inlet, $\mathrm{s} / \mathrm{L}=0.15$, 0.5. In addition, the flow rate is measured by a DFM model ultrasonic flow meter made by Greyline Company. This instrument has an accuracy of $0.01 \mathrm{lit} / \mathrm{min}$ and uses the Doppler Effect to measure the flow rate.

\section{ACOUSTIC DOPPLER VELOCIMETER AND MEASUREMENTS}

The ADV is attractive for measuring instantaneous velocities. While it is a non-intrusive remote sensing system, it can fulfill the roles of other flow meters including electromagnetic current meters, propeller meters, hot film and hot wire probes (Lohrmann et al. 1994). It is a useful tool for measuring all three components of velocity in laboratory and field environments (Best et al., 2001). This instrument is commercially available and in recent years has started to replace the previously developed flow meters.

ADV's have quickly become valuable tools for laboratory and field investigations of river flow, canals, reservoirs, oceans, and around hydraulic structures and laboratory scale models. This instrument is relatively rugged, easy to operate and can be readily mounted and maneuvered with the flow field. A $10 \mathrm{MHz}$ Nortek acoustic Doppler velocimeter was used for measuring velocities in the present experimental runs. It uses a technique known as pulse-to-pulse coherent Doppler sonar for measuring the velocity vector (Parson, 1995). The phase shift between successive backscattered signals sampled at each receiver is converted to a measurement of velocity along each beam. Factory calibration of the ADV permits conversion of along-beam velocity into an orthogonal coordinate system. The error in the prediction of mean velocities is no greater than $\pm 2.5 \mathrm{~mm} / \mathrm{s} \pm 1 \%$.

Other performance characteristics are as follows: acoustic frequency $10 \mathrm{MHz}$, velocity range $\pm 0.03,0.10,0.30,1.0$, or $2.5 \mathrm{~m} / \mathrm{s}$, velocity resolution $0.1 \mathrm{~mm} / \mathrm{s}$, velocity bias $\pm 0.5 \%$ with no measurable zero-offset in the horizontal direction, sampling rate between 0.1 and $25 \mathrm{~Hz}$, random noise approximately $1 \%$ of the velocity range at $25 \mathrm{~Hz}$ sampling rate, sampling volume less than $0.25 \mathrm{~cm} 3$, minimum distance from the sampling volume to the boundary $5 \mathrm{~mm}$ (Parson, 1995).
A fundamental advantage of the ADV is its ability to measure the flow in a small sampling volume (approximately $0.25 \mathrm{~cm}^{3}$ ) that is located 5 (or 10) cm away from the sensing elements. Therefore, while the probe is inserted into the flow, the sensing volume is several centimeters away from all physical parts of the probe, so the presence of the probe generally does not disturb the measurement. This is an important advantage for studying dense flows, because the sensing element is placed in calm water and measures the velocity of the current beneath itself.

There are three limitations when applying ADV in a sediment-laden flow (turbidity currents) that require additional considerations. Firstly, the ADV (like any acoustic sensor) measures the velocity of acoustic targets (e.g. solid particles) but not the fluid velocity. It is assumed that the sediment and the fluid travel at the same velocity. This assumption is likely to be valid only when considering fine sediment, dominantly in suspension. In our study, because the working fluid is tap water, seeding material was added to water. The second problem is the spatial changes in density and density stratification within the current and therefore changes in the acoustic velocity. Whereas the sediment concentration in the dense fluid has a value up to $3 \%$ (or $15 \mathrm{~g} / \mathrm{l}$ ) and the density of the dense fluid at this concentration is approximately $1008 \mathrm{~kg} / \mathrm{m} 3$, the change in acoustic velocity is not an inherent problem and is less than the error limits within this technique (Kawanisi, Yokosi, 1997) for these cases. Thirdly, when the sediment concentration increases up to $50 \mathrm{~g} / \mathrm{l}$, acoustic waves are absorbed in sediment-laden flow and attenuated. So the ADV cannot operate properly. The right-handed coordinate system was used in measurements and data analysis. The coordinate system is as follows: $\mathrm{x}$ positive downstream along the main flow (velocity component $\mathrm{u}$ ), y positive in the cross stream direction oriented to the left bank (velocity component $\mathrm{v}$ ), and $\mathrm{z}$ positive upward toward the water surface (velocity component w).

The instantaneous velocity recorded by ADVprobes should be checked for unreliable data and if necessary corrected before calculating the flow characteristic. Finally, corrected velocity data were used to calculate the mean flow properties. Mean velocities are calculated from the temporal averaging of the recorded time series at each point.

\section{CERTAINTY OF EXPERIMENTAL RESULTS}

To increase the physical insight into the meaning of empirical experimental results in a coherent manner, it is customary to plot the data on a graph and fit curves to quantify the scatter of data and determine whether any special trend exists.

A polynomial in order $n$ is fitted to the data points to establish the trend of the velocity profile in each section. Figure 3a illustrates typical experimental data and standard deviations at $x / L=0.82$ when the baffle is absent (test case $1)$. The following relation is then used as the best estimate for standard deviation $\sigma$ (Nikoran et al., 2002) 


$$
\sigma=\left[\frac{1}{n-1} \sum_{i=1}^{n}\left(u_{i}-\bar{u}\right)^{2}\right]^{1 / 2}
$$

In which $\bar{u}$ is the average velocity in $x$ direction along the channel and $u_{i}$ is the measured velocity in each experiment and was calculated as follows

$$
\bar{u}=\frac{1}{n} \sum_{i=1}^{n} u_{i}
$$

The obtained data are depicted in Fig. 3b. A greater standard deviation $\sigma$ means more uncertainty in the results. The systematic and random errors being associated with the measurement in experiment or instrumentation, or both, must be analyzed for a complete correction or explanation. It should be noted that temperature difference due to temperature fluctuations and the high thermal capacity of water are major sources of errors in this experiment. As a result of these phenomena a jet of flow generates at the bed of the tank. In general, uncertainty is greater near the bottom of the channel and also on the free surface.

\section{EXPERIMENTAL RESULTS}

Figure 4 demonstrates the variation of the velocity profiles along the primary settling tank. The trends of the laboratory results studied by the interpolation method discussed in the previous section were shown by solid lines. In test case 1 , as it can be observed from Fig. $4 \mathrm{a}$, an inlet jet exists in the first section of the basin; $x / L=0.44$ and can deflect the velocity profile at the free surface.

This current begins near the bottom. The declination of the velocity at the upper height of the tank divulges the existence of a circulation region near the free surface of water. Before reaching $x / L=0.44$, water escapes to the outlet as a result of the existing short circuit regime. As water flows downstream, the maximum velocity is at the bottom and shifts toward the upper parts, near the free surface and overflows. This phenomenon can be observed in $x / L=0.56$ (Fig. 4a). As it was expected, when the velocity profile becomes uniform at a certain part of the channel $x / L=0.69$, the sedimentation retention increases. Additionally, the jet moves upward and its changes are considerable at $x / L=0.56$. Furthermore, the velocity decreases at the surface at $x / L=0.69$ as results depict. The maximum velocity is increased at $x / L=0.82$ and is prone to rise up. In this section As a result of the existence of the overflow and effluent effect, a reverse flow occurs near the free surface influenced by the flow circulation.

In continuation, it can be seen from Fig. $4 b$ \& 4c that the entering water quality is very sensitive to the tank geometry. Examination of velocity magnitudes does, however, reveal a possible explanation for the hysteresis. In test case 2 (Fig. $4 \mathrm{~b}$ ), the existence of the baffle at the vicinity of the channel inlet leads to the dissipation of the kinetic energy, a decrease in maximum magnitude of the stream-wise velocity and the upward inclination of the velocity field in $x / L=0.44$. Moreover, the downstream circulation of the baffle inclines the velocity profile more to the surface at this section. In the absence of the bottom jet and the viscous effects, the momentum of the particles enhances. This momentum increase in vertical direction improves the sedimentation conditions. Consequently, this upward trend of the velocity profile provides a better chance for particles to settle down in this section. In the section $x / L=0.82$ the flow current moves upward due to the effluent in end of the sedimentation basin.

Test case 3 elucidates the experimental results in the existence of the baffle in the middle of the tank. Hence, the baffle was placed at $0.5 \mathrm{~L}$. Right at the baffle location, due to the reduction of cross section area, the velocity increases and its effect is observed at $x / L=0.56$. After the baffle, a large recirculation region exists which deteriorates the uniform condition. This phenomenon mixes the fluid flow once more as the inclination of the graph near the bottom explains. Thus, the baffle not only weakens the proper condition for particle deposit but also worsens it. Furthermore, the maximum magnitude of velocity is raised in this location of the baffle. This phenomenon increases the kinetic energy in the flow field especially after baffle at $x / L=0.56$. The velocity profiles in other sections of the tank demonstrate a similar trend to the previous cases. In this case, it seems that two recirculation regions exist in the tank and the volume of the dead zones increases.

\section{MAThematical Modeling}

\subsection{Governing Equations}

In order to obtain a precise model, the 2-D two-phase airwater equations are considered with an Eulerian-Eulerian method. In this study, the equations for both fluids will be coupled with another equation which relates the volume fraction of two phases at the interface. The fields for all variables and properties are shared by the phases and represent volume-averaged values, as long as the volume fraction of each of the phases is known at each location. Thus, the variables and properties in any given cell are either purely representative of one of the phases, or representative of a mixture of the phases, depending upon the volume fraction values. The governing equations are the conservation of mass and momentum. For closing the equations of motion, a turbulence model is added to these equations to calculate the Reynolds stresses. This study has been carried out by FLUENT Software (Fluent Manual), version 6.1 to solve numerical equations. Moreover, grids are generated by Gambit Software to discretize the physical domain . The mass conservation equation for each phase is

$$
\frac{D \rho}{D t}+\rho(\nabla . V)=0
$$

A single momentum equation is solved throughout the domain, and the resulting velocity field is shared among the phases. The momentum equation, shown below, is dependent on the volume fractions of all phases through the properties $\rho$ and $\mu$.

These equations are written with the Bousinesq approximation. In other words, if the qth fluid's volume fraction in a cell is denoted as $\alpha_{q}$, then the following three 
conditions are possible: the cell is empty $\alpha_{q}=0.0$, (of the qth fluid), the cell is full, $\alpha_{q}=1.0$, the cell contains the interface between the qth fluid and one or more other fluids, $0<\alpha_{q}<1$, (Sadathosseini et al., 2007).

$$
\begin{aligned}
& \frac{\partial u}{\partial t}+u \frac{\partial u}{\partial x}+v \frac{\partial u}{\partial y}=-\frac{1}{\rho} \frac{\partial p}{\partial x}+\frac{\partial}{\partial x}\left(\left(v+v_{t}\right) \frac{\partial u}{\partial x}\right) \\
& +\frac{\partial}{\partial y}\left(\left(v+v_{t}\right) \frac{\partial u}{\partial y}\right) \\
& \frac{\partial v}{\partial t}+u \frac{\partial v}{\partial x}+v \frac{\partial v}{\partial y}=-\frac{1}{\rho} \frac{\partial p}{\partial y}+\frac{\partial}{\partial x}\left(\left(v+v_{t}\right) \frac{\partial v}{\partial x}\right) \\
& +\frac{\partial}{\partial y}\left(\left(v+v_{t}\right)+\frac{\partial v}{\partial y}\right)
\end{aligned}
$$

Based on the local value of $\alpha_{q}$, the appropriate properties and variables will be assigned to each control volume within the domain. The tracking of the interface between the phases is accomplished by the solution of a continuity equation for the volume fraction of one of the phases. For the $\mathbf{q}_{\mathrm{th}}$ phase, this equation has the following form in this study with $\mathbf{q}=2$.

$$
\frac{\partial \alpha_{q}}{\partial t}+\vec{v} \cdot \nabla \alpha_{q}=0
$$

The volume fraction equation will not be solved for the primary phase; the primary-phase volume fraction will be computed based on the following constraint:

$$
\sum_{1}^{n} \alpha_{q}=1
$$

Air-water phases and the interface are properly modeled by the VOF multiphase technique.

\subsection{Turbulence Model}

Although the Reynolds number of this flow is rather low, the flow is turbulent when entering the channel. In addition, the flow field contains curvature streamlines and causes intensive anisotropy in flow fields. Thus, using the standard $k-\varepsilon$ model, the true size of the recirculation region cannot be perceived. In the previous studies (Tamayol, Firoozabadi, 2006), we have shown that the $k-\varepsilon$ RNG model can capture the curvature of the streamlines better than the standard $k-\varepsilon$ model. Then, the $k-\varepsilon$ RNG model is used for turbulence modeling here. The corresponding transport equations for turbulent kinetic energy (k) and its dissipation rate $(\varepsilon)$ are (Yakhot, Orszag, 1986)

$\rho \frac{D k}{D t}=\frac{\partial}{\partial x_{i}}\left[\alpha_{k} \mu_{e f f} \frac{\partial k}{\partial x_{i}}\right]+G_{k}+G_{b}-\rho \varepsilon-Y_{M}$

$$
\begin{aligned}
& \rho \frac{D \varepsilon}{D t}=\frac{\partial}{\partial x_{i}}\left[\alpha_{k} \mu_{e f f} \frac{\partial \varepsilon}{\partial x_{i}}\right]+C_{1 \varepsilon} \frac{\varepsilon}{k}\left(G_{k}+C_{3 \varepsilon} G_{b}\right) \\
& -C_{2 \varepsilon} \rho \frac{\varepsilon^{2}}{k}-R
\end{aligned}
$$

In these equations, $G_{b}, Y_{M}$, and $G_{k}$ are production terms of turbulence kinetic energy due to buoyancy, compressibility and mean velocity gradients, respectively. The first two, here are zero but the third is

$$
G_{k}=-\rho \overline{u_{i}^{\prime} u_{j}^{\prime}} \frac{\partial u_{j}}{\partial x_{i}}
$$

Model constants are: $C_{1 \varepsilon}=1.42, C_{2 \varepsilon}=1.68$. Although the turbulent viscosity can be computed from a differential equation, an algebraic relation is used. i.e,

$$
\mu_{t}=\rho C_{\mu} \frac{k^{2}}{\varepsilon}, C_{\mu}=0.0845
$$

In Eq. 10, $R$ is defined as:

$$
\begin{aligned}
R & =\frac{C_{\mu} \rho \eta^{3}\left(1-\eta / \eta_{0}\right)}{1+\beta \eta^{3}} \frac{\varepsilon^{2}}{k} \\
S & =\left[\frac{1}{2}\left(\frac{\partial u_{i}}{\partial x_{j}}+\frac{\partial u_{j}}{\partial x_{i}}\right)\left(\frac{\partial u_{i}}{\partial x_{j}}+\frac{\partial u_{j}}{\partial x_{i}}\right)\right]^{\frac{1}{2}}
\end{aligned}
$$

Since

$$
\eta=\frac{S k}{\varepsilon}, \eta_{0}=4.38, \beta=0.012
$$

\subsection{Boundary and Initial Conditions}

At the rigid walls, no slip conditions are applied. But, applying these boundary conditions, the real data may not be captured. In the present study, another condition is used in order to gain the results that agree with experiments precisely. The flow and the turbulent equations have to be accurately resolved to obtain the velocity distribution predictions. All computations were performed in Cartesian coordinates with rectangular geometry. Rectangular grids were used with a high resolution near all solid boundaries and the intefaces between two phases. In all cases, the inlet velocity is assumed uniform, and the solutions presented here are considered grid independent. In this work, a wall function with the following equation is applied (Sadathosseini et al., 2007)

$$
u_{\tau}=\frac{\overline{u_{p}} \kappa}{\ln \left(\mathrm{E} y^{+}\right)}
$$

where $u_{\tau}=\sqrt{\tau_{w} / \rho}$ is the friction velocity, $\left(\tau_{w}\right.$ is the shear stress at the wall), $\bar{u}_{p}$ is the velocity in the direction of boundary at the first node above the wall surface and $y^{+}=u_{\tau} y / v$ is the non-dimensional distance from the 
wall. In this relation the Von-Karman constant $\kappa=0.41$ and the roughness of the wall is $E=9.8$. For the case $y^{+}<11.6$ the amplitudes of $\kappa, u_{\tau}, \varepsilon$ are obtained from interpolation and constrains of viscose sub-layer.

In the present work, the value of the flow rate is set at 42 lit/min similar to the laboratory experiments., In the numerical method, initially the channel is considered to be full of air (one phase). Water enters the tank uniformly and replaces the first phase in the tank. The water level in the tank increases until it overflows at the end of the channel through a weir.

A finite volume approach is used for the solution of the governing equations. Second order upwind scheme is selected to discretize the governing equations. SIMPLEC algorithm is used for pressure-velocity coupling.

Extensive tests were undertaken to establish a gridindependent solution. More than $680 * 53$ mesh points were required before the velocity contours changed to independent grid; then, $680 * 53$ grids were chosen for the computation. Sampling of the numerical grids used for prediction of the settling tank for test case 1 is shown in Fig. 5.

\section{NUMERICAL RESULTS}

\subsection{Validation}

The velocity profiles achieved from VOF method in comparison with the experimental results are shown in the following Figures. Figure 6 shows test case 1 which is the absence of the baffle case. In this figure, solid lines are numerical results.

It is observed that the numerical data match the laboratory results very well. But near the surface and close to the bed, some errors were observed. As we previously mentioned, each experiment was repeated 6 times. Then, one of the sources of errors is probably ADV. This device can detect the velocity of the small particles in about $5 \mathrm{~cm}$ below of the receiver part of ADV. Three-dimensional effects are also pointed out as other sources of the errors. We have examined other turbulence models (such as standard $k-\varepsilon$ model) and simulation methods such as the single-phase flow, but the two-phase flow method agrees with experimental data better than the other solutions. Present simulation was also verified by comparing the experimental data when a baffle exists. Figure 7 shows the calculation results compared with the experimental data in test case 3 , in which a baffle was placed at $\mathrm{s} / \mathrm{L}=0.5$. It is seen that the agreement between the present model and the experimental data is also good, while baffle exists and the flow field becomes more complicated.

After analyzing the graph, as we discussed in the previous section, a circulation region as well as a jet at the inlet can be observed in this case. This pattern has a magnitude of kinetic energy, which seems to have been dissipated to prevent chaotic behavior near the bed and provide a better condition for sedimentation. In experiments, for finding the best location of the baffle, the laboratory experiments should have been repeated many times. That expends the time and is a hard process, so for distinguishing the best situation for the baffle, the present numerical model was developed.

\subsection{Discussion}

In this study, after verification of the present model, the best baffle location was found out. In this approach, the best location of the baffle is obtained when the volume of the circulation zone is minimized or the dead zone is divided into smaller parts. The circulation volume which is normalized by the total water volume in the tank and calculated by the numerical method in different baffle locations is shows in Table 1. From this table, it is predictable that some cases must have had poor performances. This is related to the size of dead zone. When we use a baffle in the tank, three circulation regions may appear whose size is sensitive to the position of the baffle.

If we can infer that the optimal baffle creates the smaller circulation volume, Table 1 shows that the baffle performance at $s / L=0.125$ is the best. For increasing the performance of the settling tanks this method has been suggested, which is to use the grid near the inlet. This kind of baffle may lead to a more uniform distribution of velocity in the tank and minimizes the dead zones. This table also shows that the baffle located at the end part of the channel is worse than no-baffle condition. For better judgment different positions of the baffle were examined numerically.

Different positions of the baffle near the inlet, present the same results (Table 1 ). These values must be compared with the percentage volume of 14.24 occupied by the circulation region existing in the tank without baffle. A smaller amount of circulation appears to be in $\mathrm{s} / \mathrm{L}=0.125$.

Figure 8 shows the streamlines of the optimal baffle location. As it is observed, two circulation regions exist in the tank. In spite of these regions, the circulation volume is minimized and it seems this baffle divides the dead zone into two parts. The turbulent kinetic energy of this configuration is then examined to secure the optimal position.

The present results from numerical simulation provide in Fig. 9; there is a comparison between the characteristics such as velocities in $\mathrm{x}(\mathrm{U})$ and $\mathrm{y}(\mathrm{V})$ directions, and turbulent kinetic energy of the case (baffle at $\mathrm{s} / \mathrm{L}=0.125$ ) and in case (the absence of the baffle). Two left columns depict the velocity profiles of these cases at different locations of the tank.The inlet jet near the bed in case $\mathrm{s} / \mathrm{L}=0.125$ is deteriorated. The behavior of $U$ profile has a tendency to be more monotonous than the case1. Moreover, the maximum velocities in this case reduced. In addition velocity profiles (left column of Fig. 9) has a smaller slope on the bed which means that after the baffle the shear stress reduces on the bed. The flow has a trend to be calm at the remaining part of the channel so that the baffle can make a mixing current in the flow field right after passing it.

A common behavior near the velocity $\mathrm{V}$ is found in both cases except at the inlet. For case $s / L=0.125$ the return flow 
from surface to the bed observed in $\mathrm{x} / \mathrm{L}=0.44$. Moreover its value decays to the end of the basin.This numerical outcome is based on the Mass-Weighted-Turbulence kinetic energy. The results indicate that, although this factor increased in the presence of the baffle, it can be reduced at the next stations. It is expected that this baffle can create a calm condition to settle down the particles.

A more direct comparison of the mass weighted average of turbulent kinetic energy $k$ in various cases of flow is afforded by Table 2; this factor has a range of value for different baffle position. Reduction of turbulent kinetic energy for the optimal baffle case $(\mathrm{s} / \mathrm{L}=0.125)$ is shown in this table. Furthermore, the streamlines of each case are shown in Fig. 10. The dead zones can be observed from the figure.

Additionally, the formation of circulation regions reduced the efficiency of the sedimentation tank by short circuiting and this negative phenomenon is improved by proper position of the baffle. In other words, locating a baffle at an appropriate part of the tank prevents from formation the bottom jet moving to the surface of the basin and overflows at the outlet.

\section{CONCLUSION}

Settling tanks have been used for separating floating particles from the main flow. To optimize the operation of these tanks, it is required that calm flow of fluid be formed in the tank in such a way that the sedimentation process is performed in a best way. However, the creation of the dead zone and short circuiting inlet to the outlet disturbs the calmness of the flow and leaves negative influences on the performance of the tank. Settling tanks are divided into two main categories in type and concentration of sludge and particles which are available in them. Primary settling tanks have low influent concentration. Then, from a hydraulic point of view, the presence of particles does not affect the flow field and they can be investigated without particles. In fact, many factors influence the tank's efficiency, amongst which the tank geometries is of primordial importance.

In this work, the experimental and numerical approaches were performed to investigate the effects the baffle position on the flow field. In laboratory, a test rig was conducted to find the effect of the baffle position on the velocity profiles by ADV. Then, using CFD and VOF method, a numerical simulation of flow in the tank was developed by Fluent software. Using the experimental data, the numerical results were verified. Finally, the optimal location of the baffle was found numerically. Results show that this baffle can reduce the size of the dead zones and turbulent kinetic energy in comparison with the no-baffle condition.

\section{REFERENCES}

Adams, E.W. and W. Rodi (1990). Modeling flow and mixing in sedimentation tanks. J. of Hydraulic Engineering 116 (7), 895-913.

Armenio, V. and M. LaRocca (1996). On the analysis of sloshing of water in rectangular containers: Numerical study and experimental validation. Ocean Engineering 23(8), 705-739.

Ashjari, M.A. and B. Firoozabadi (2003). Using the nonlinear $k-\varepsilon$ in calculation of performance of settling tanks. ISME, 11th Int. Conf. Mech. Engineering, Mashhad, Iran.

Best, J., A.D. Kirkbride, and J. Peakall (2001). Mean flow and turbulence structure of gravity currents. Sediment transport and deposition by particulate. Spec. Pub., 159-173.

Brescher, U., Krebs, P. and W.H. Hager (1992). Improvement of Flow in Final Settling Tanks. Journal of Environmental Engineering 188, 307-321.

Celik, I., Rodi, W. and A.I. Stamou (1985). Prediction of hydrodynamic characteristics of rectangular settling tanks. Int. Symposium of Refined Flow Modeling and Turbulence Measurements, Iowa, USA.

FLUENT, Version 6.1, User Manual, Fluent Inc.

Fugate, D.C. and C.T. Fredrichs (2002). Determining concentration and fall velocity of estuarine particle populations using ADV. OBS and LISST, Cont. Shelf Res. 22, 1867-1886.

Hay, A.E. (1983). On the remote acoustic detection of suspended sediment at long wavelengths. J. Geophys. Res. 88, 7525-7542.

Krebs, P. (1995). Success and shortcomings of clarifier modeling. Journal of Water Science and Technology 31(2), 181-191.

Kawanisi, K. and S. Yokosi (1997). Measurements of suspended sediment and turbulence in tidal boundary layer. Cont. Shelf Res. 17, 859-875.

Libicki, C., K.W. Bedford, and J.F. Lynch (1989). The interpretation and evaluation of a $3-\mathrm{MHz}$ acoustic backscatter device for measuring benthic boundary layer sediment dynamics. J. Acoust. Soc. Am. 85, 1501-1511.

Lohrmann, A., R. Cabrera, and N.C. Karus (1994). Acoustic Doppler velocimeter (ADV) for laboratory use. In Proc. Symposium on Fundamental and Advancements in Hydraulic Measurements, A.S.C.E., New York, 351-365.

Lyn, D.A., A.I. Stamou, and W. Rodi (1992). Density currents and shear-induced flocculation in sedimentation tanks. J. of Hydraulics Eng., ASCE 118(6), 849-867.

Matko, T., N. Fawcett, A. Sharp, and T. Stephenson (1996). Recent progress in the numerical modeling of 
wastewater sedimentation tanks. Process Safety and Environmental Protection 74(B4), 245-258.

Nikora, V.I., D.G. Goring, and A. Ross (2002). The structure and dynamics of the thin near-bed layer in a complex marine environment. Estuarine, Coastal Shelf Sci. 54, 915-926.

Nortek (1999). ADV Specification User and Operation Manual.

Parsons, J.D. (1998). Mixing mechanisms in density intrusions. Ph.D. Thesis, University of Illinois at Urbana-Champaign.

Robert, J.M. (1988). Describing the Uncertainties in Experimental Results. J. Experimental Thermal fluid Science 1, 3-17.

Rodi, W. (1993). Turbulence Models and their Application in Hydraulics. IAHR, Delft, The Netherlands.

Sadathosseini, S. H., S.M. Mousaviraad, B. Firoozabadi, and G. Ahmadi (2007). Numerical Simulation of Free Surface Waves and Wave Induced Separation. Scientia of Iranica Journal (accepted for publication).
Stamou, A.L., E.W. Adams, and W. Rodi (1990). Numerical modeling of flow and settling in primary rectangular Clarifiers. J. of Hydraulic Research 27, 665-682.

Taebi-Harandy, A. and E.D. Schroeder (1995). Analysis of structural features on performance of secondary clarifiers. Journal of Environmental Engineering 121(12), 911-919.

Tamayol, A., and B. Firoozabadi (2006). Effects of turbulent models and baffle position on hydrodynamics of settling tanks. Scientia of Iranica Journal 13(3), 255-260.

Tamayol, A., B. Firoozabadi, and G. Ahmadi (2006). Increasing performance of final settling tanks by using baffles. $7^{\text {th }}$ International Conference on Hydroinformatics, HIC 2006, Nice, France.

Tamayol, A., B. Firoozabadi, and G. Ahmadi (2007). Effects of inlet position and baffle configuration on the hydraulic performance of primary settling tanks. Journal of Hydraulic Engineering, ASCE (accepted for publication).

Yakhot, V. and S.A. Orszag (1986). Renormalization Group Analysis of Turbulence, I. Basic Theory. Journal of Scientific Computation 1(1), 1-51.

Table 1 Circulation volume percentage in a different position of the baffle

\begin{tabular}{|c|c|c|c|c|c|}
\hline s/L & 0.1 & $\mathbf{0 . 1 2 5}$ & $\mathbf{0 . 1 5}$ & $\mathbf{0 . 5}$ & $\begin{array}{c}\text { No } \\
\text { baffle }\end{array}$ \\
\hline CV (\%) & 13.28 & 13.05 & 13.61 & 17.48 & 14.24 \\
\hline
\end{tabular}

Table 2 Mass weighted average of turbulent kinetic energy $\mathrm{k}$ in $\mathrm{x} / \mathrm{L}$ sections

\begin{tabular}{|c|c|c|c|c|}
\hline $\boldsymbol{x} / \mathbf{L}$ & 0.44 & 0.56 & 0.69 & 0.82 \\
\hline $\boldsymbol{s} / \mathbf{L}=\mathbf{0 . 5}$ & $8.7 \mathrm{E}-6$ & $1.05 \mathrm{E}-5$ & $1.74 \mathrm{E}-5$ & $3.71 \mathrm{E}-5$ \\
\hline $\boldsymbol{s} / \mathbf{L}=\mathbf{0 . 1 5}$ & $6.8 \mathrm{E}-6$ & $3.6 \mathrm{E}-6$ & $4.7 \mathrm{E}-6$ & $3.9 \mathrm{E}-6$ \\
\hline $\boldsymbol{s} / \mathbf{L}=\mathbf{0 . 1 2 5}$ & $6.6 \mathrm{E}-6$ & $1.9 \mathrm{E}-6$ & $4.0 \mathrm{E}-6$ & $1.1 \mathrm{E}-6$ \\
\hline $\boldsymbol{N o}$ baffle & $1.6 \mathrm{E}-6$ & $3.6 \mathrm{E}-6$ & $4.8 \mathrm{E}-6$ & $3.91 \mathrm{E}-6$ \\
\hline
\end{tabular}

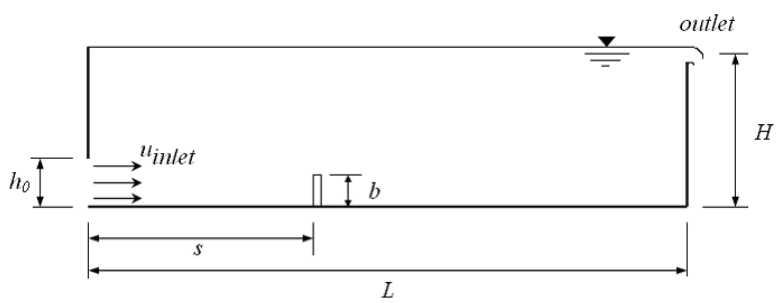

Fig. 1. Schematic diagram of the tank, $h_{0}=11 \mathrm{~cm}, b=8 \mathrm{~cm}$, $\mathrm{H}=34 \mathrm{~cm}$, and $\mathrm{L}=800 \mathrm{~cm}$.
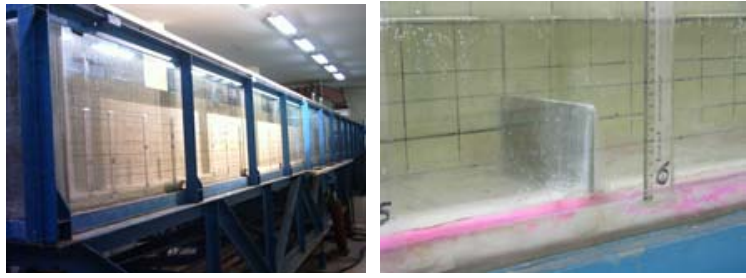

Fig. 2. Photos of laboratory setup, a) Settling tank (left), b) Baffle (right). 
A. Razmi et al. / JAFM , Vol. 2, No. 1, pp. 1-12, 2009.
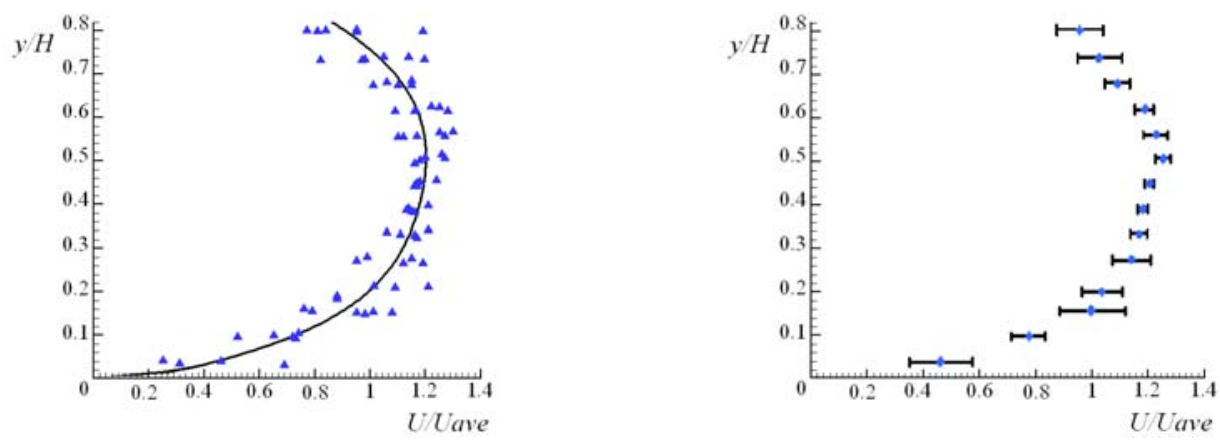

Fig. 3. a) The curve is fitted (solid line) to the measured data (triangles) at $x / L=0.82$ in case1, b)The standard deviation of results.

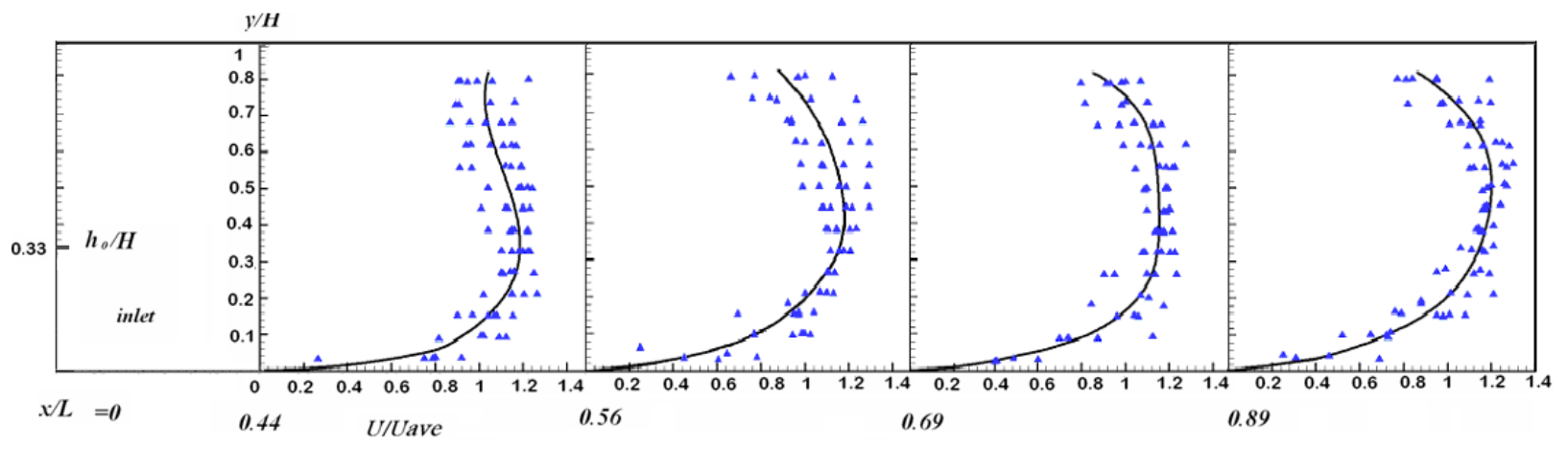

a) No-Baffle (test case 1)

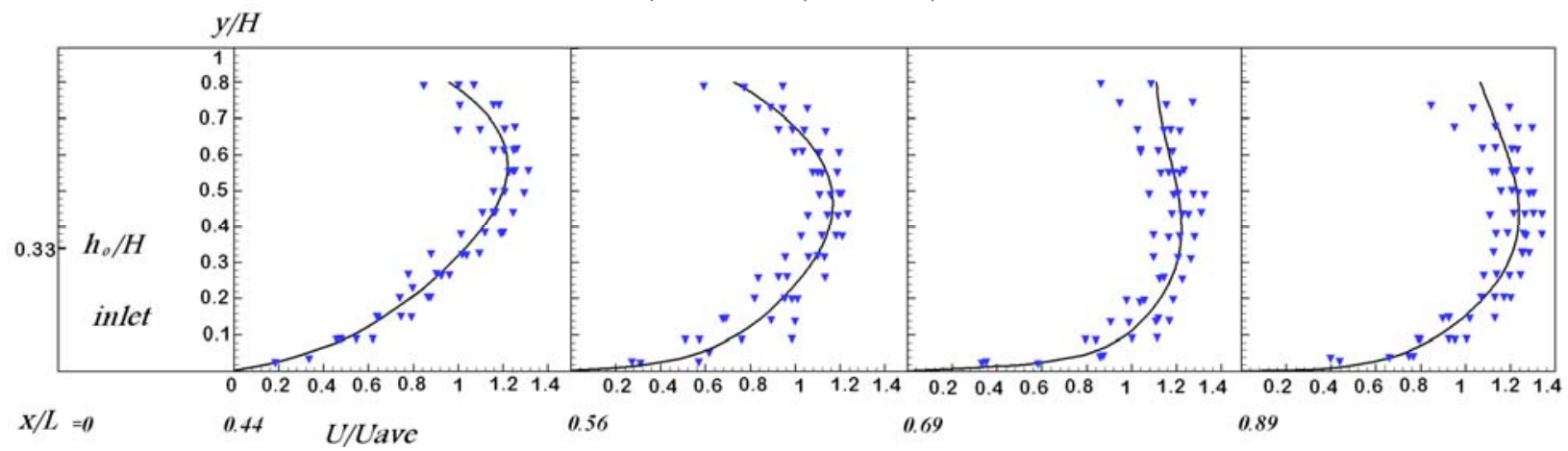

b) Baffle at $0.15 \mathrm{~L}$ (test case 2)

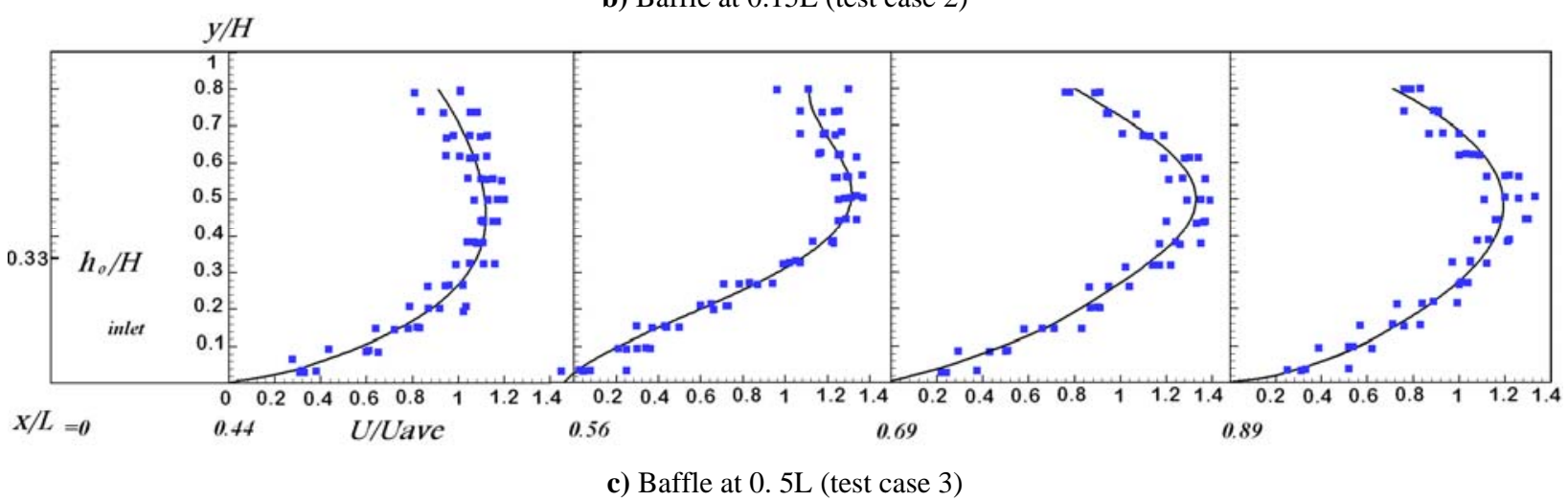

Fig. 4. Stream-wise velocity profiles in the tank in different location of test cases: 1, 2, 3. 
A. Razmi et al. / JAFM , Vol. 2, No. 1, pp. 1-12, 2009.

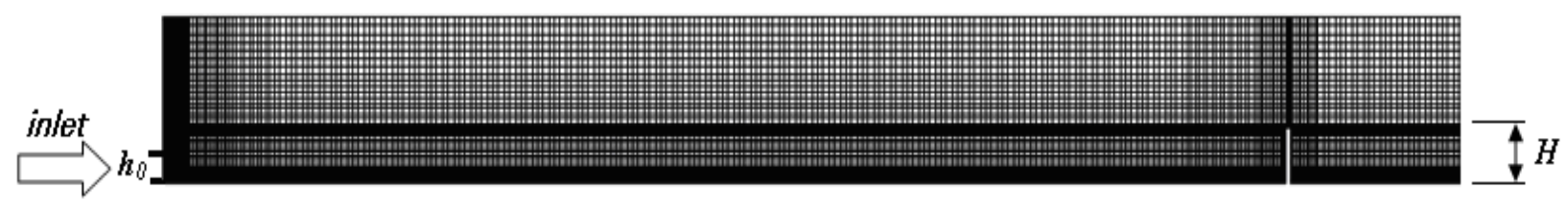

Fig. 5. Sampling of numerical grids used for prediction basin case1 (No-Baffle)

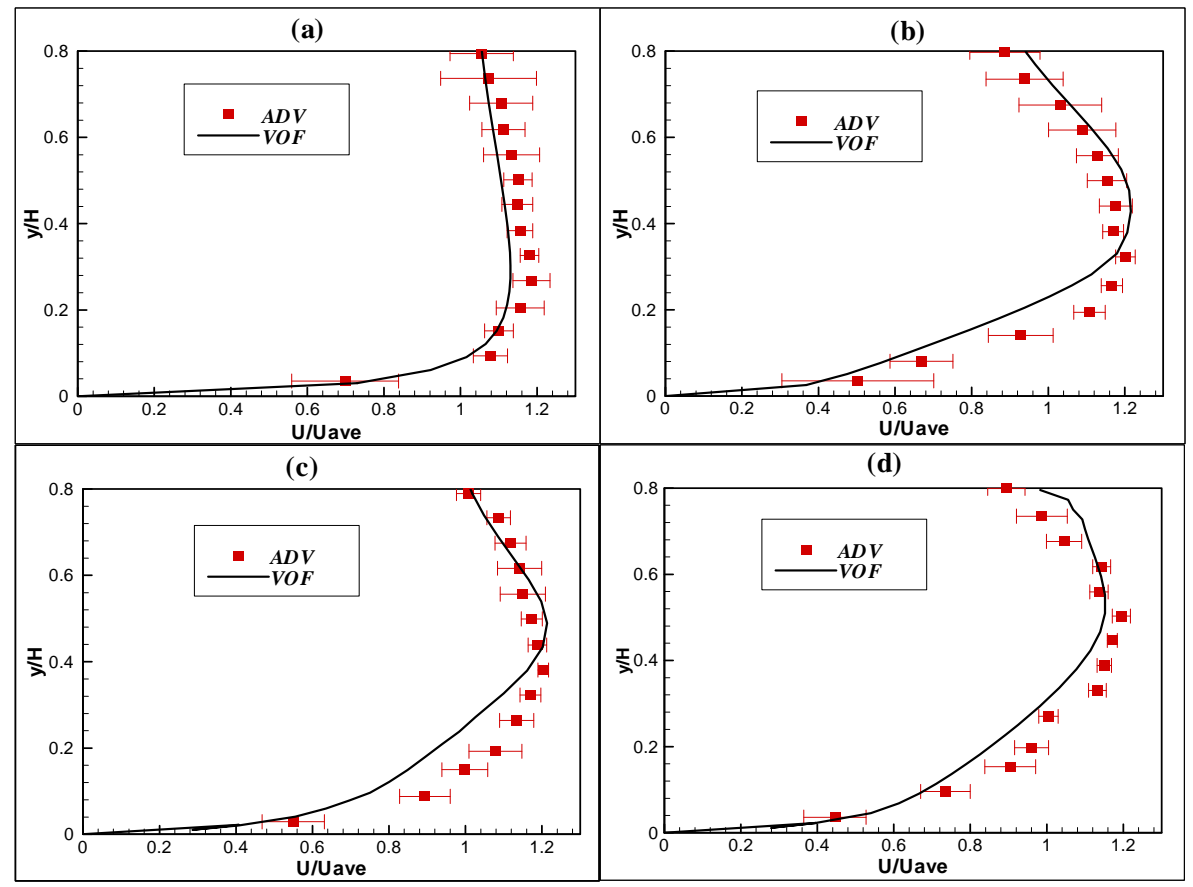

Fig. 6. The comparison between numerical and experimental results in test case $1 \mathrm{a}) \mathrm{x} / \mathrm{L}=0.44, \mathrm{~b}) \mathrm{x} / \mathrm{L}=0.56, \mathrm{c}) \mathrm{x} / \mathrm{L}=0.69$, d) $\mathrm{x} / \mathrm{L}=0.82$

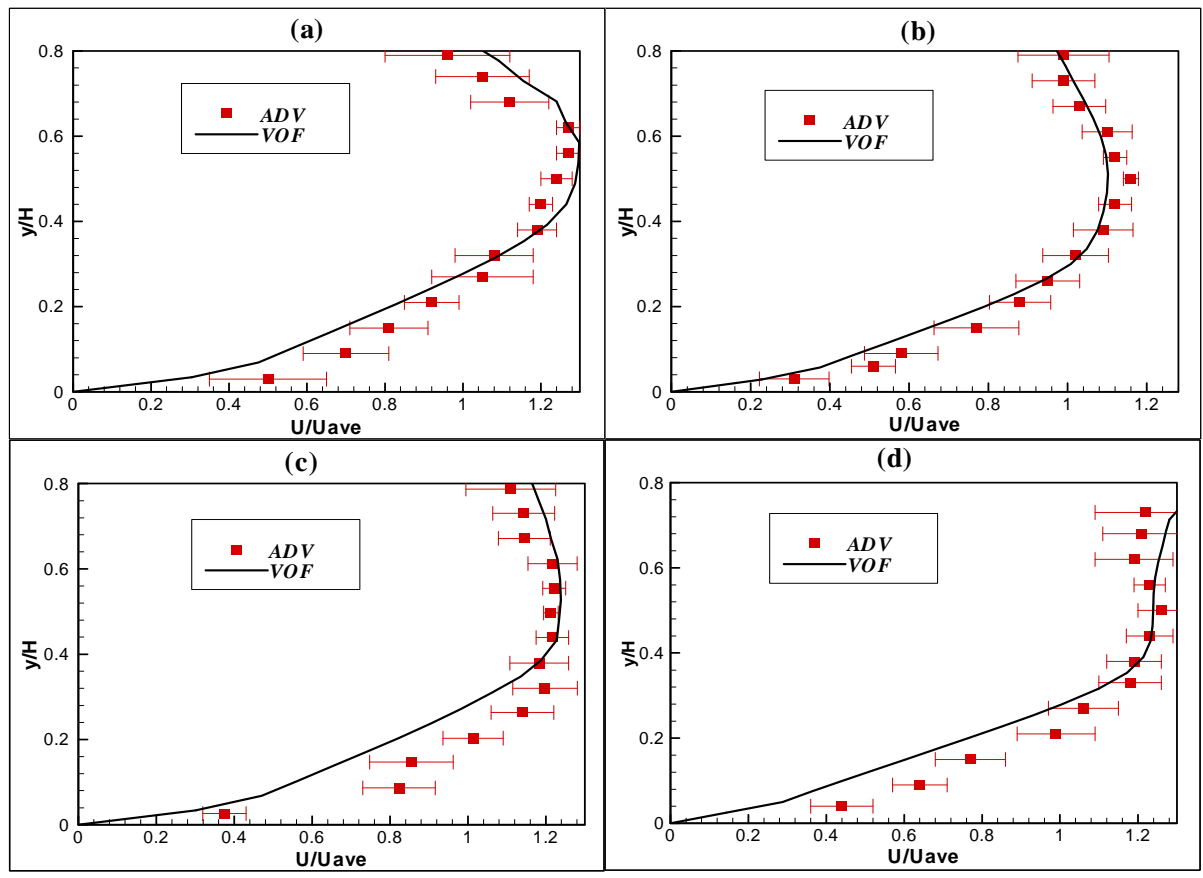

Fig. 7. Comparison between numerical method and experimental data in test case 3 .

a ) $\mathrm{x} / \mathrm{L}=0.44, \mathrm{~b}) \mathrm{x} / \mathrm{L}=0.56$, c) $\mathrm{x} / \mathrm{L}=0.69$, d) $\mathrm{x} / \mathrm{L}=0.82$ 
A. Razmi et al. / JAFM , Vol. 2, No. 1, pp. 1-12, 2009.

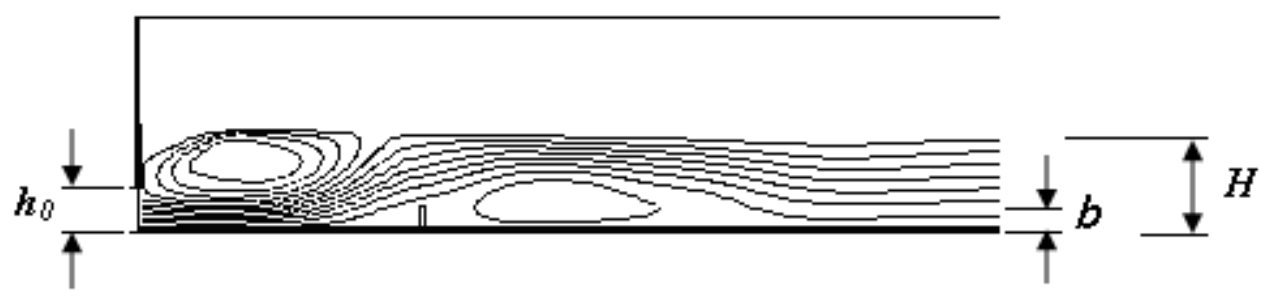

Fig. 8. A baffle at $1.2 \mathrm{~m}$ divides the dead zone. b, $\mathrm{H}$ and $\mathrm{h} 0$ are baffle height, the surface level height, and iterance height

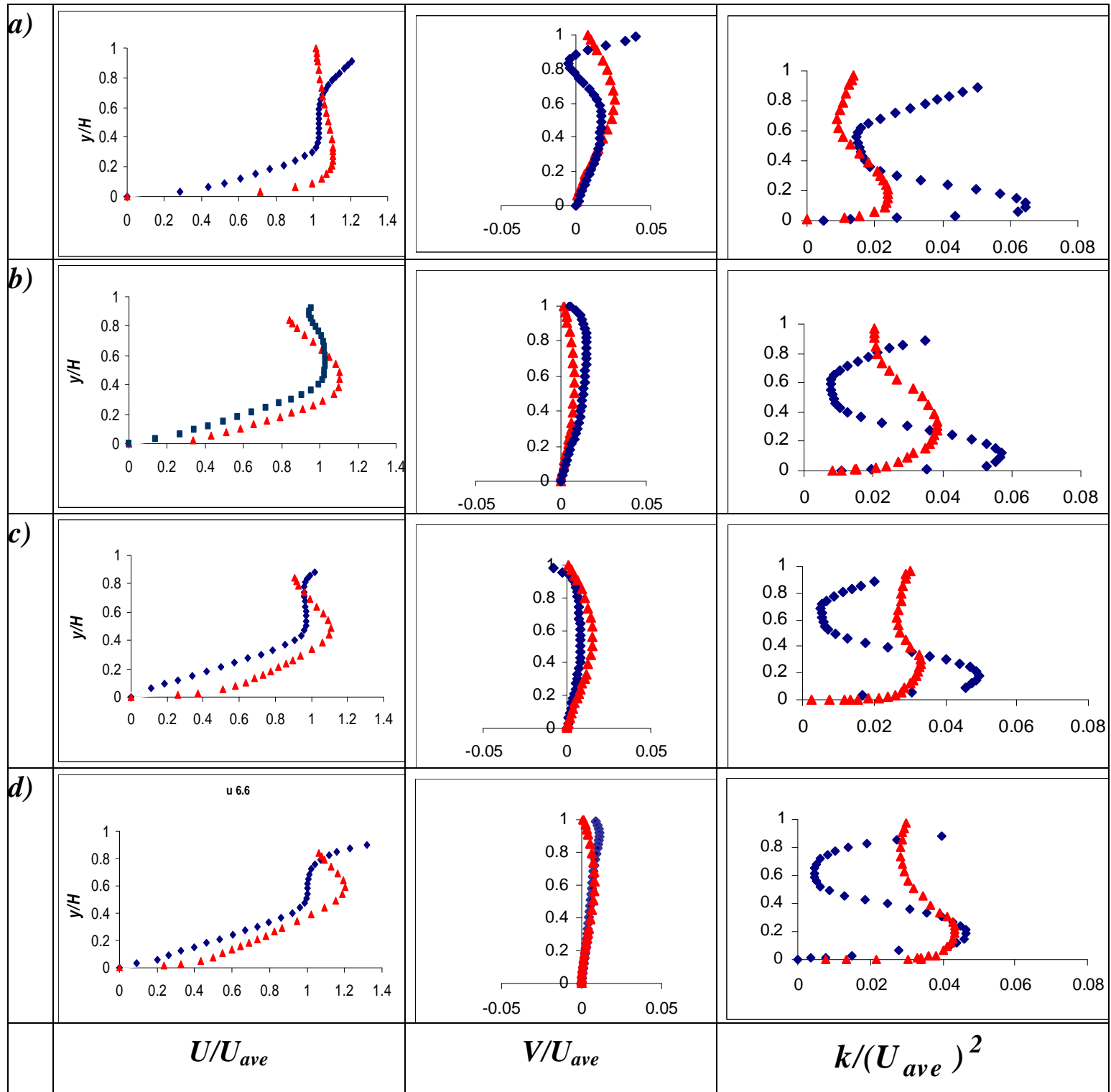

Fig. 9. Numerical simulation results of (VOF), V/Uave, U/Uave, $k /\left(U_{\text {ave }}\right)^{2}$ profiles - Comparing no baffle (trianglered) and baffle at $\mathrm{s} / \mathrm{L}=0.125$ (1.0 $\mathrm{m}$ from the inlet) (tetra angel- blue). 
A. Razmi et al. / JAFM , Vol. 2, No. 1, pp. 1-12, 2009.

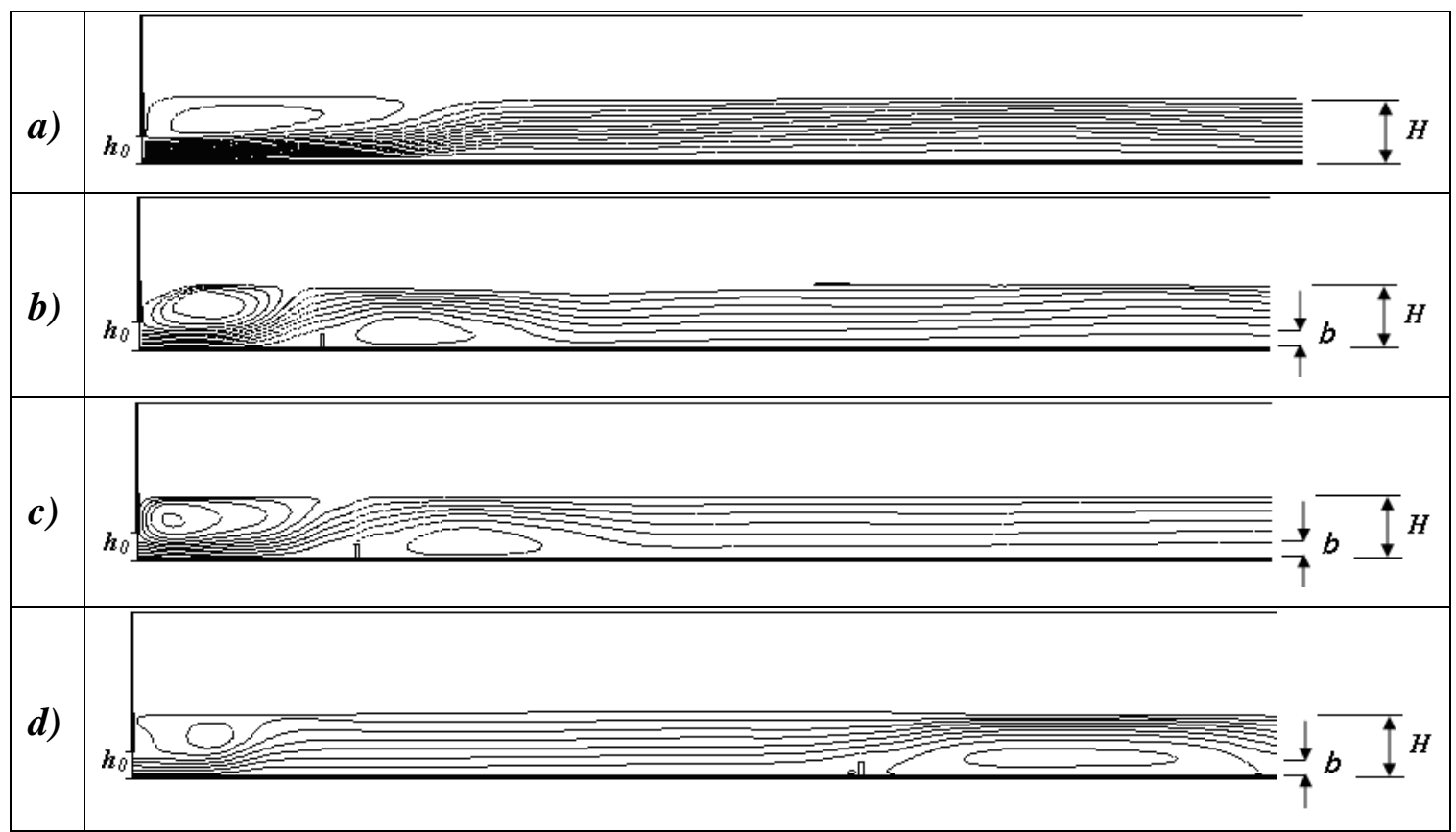

Fig. 10. Streamlines of different baffle locations in settling tank from above: a) No baffle, b) s/L=0.125,

c) $\mathrm{s} / \mathrm{L}=0.15$, d) $\mathrm{s} / \mathrm{L}=0.5$. 